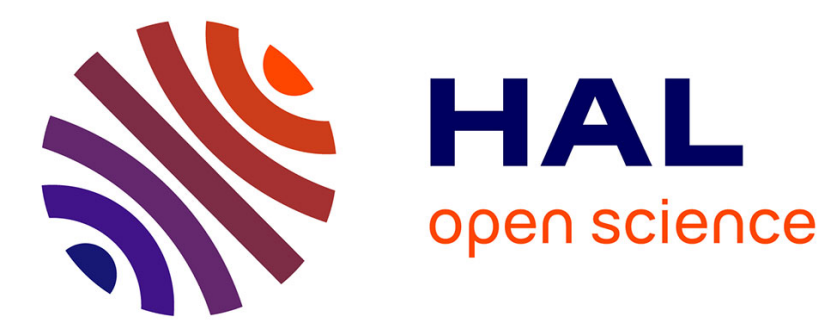

\title{
Function Decomposition in Main and Lesser Peaks
}

Robin Alais, Petr Dokládal, Etienne Decencière, Bruno Figliuzzi

\section{To cite this version:}

Robin Alais, Petr Dokládal, Etienne Decencière, Bruno Figliuzzi. Function Decomposition in Main and Lesser Peaks. International Symposium on Mathematical Morphology, May 2017, Fontainebleau, France. 10.1007/978-3-319-57240-6_26 . hal-01432417

\section{HAL Id: hal-01432417 https://hal.science/hal-01432417}

Submitted on 11 Jan 2017

HAL is a multi-disciplinary open access archive for the deposit and dissemination of scientific research documents, whether they are published or not. The documents may come from teaching and research institutions in France or abroad, or from public or private research centers.
L'archive ouverte pluridisciplinaire HAL, est destinée au dépôt et à la diffusion de documents scientifiques de niveau recherche, publiés ou non, émanant des établissements d'enseignement et de recherche français ou étrangers, des laboratoires publics ou privés. 


\title{
Function Decomposition in Main and Lesser Peaks
}

\author{
Robin Alais, Petr Dokládal, Etienne Decencière, and Bruno Figliuzzi \\ PSL Research University - MINES ParisTech, CMM, Center for Mathematical Morphology \\ 35 rue Saint Honoré - Fontainebleau, France
}

\begin{abstract}
This article shows how the dynamics extinction value can be used to compute the decomposition of a function as a sum of simpler components. We show that this decomposition induces a hierarchical segmentation of the domain of definition, and a new partial ordering on nonnegative functions. Removing some of the components according to different criteria leads to new morphological operators. Their properties are discussed and illustrated in the last section. In particular, we see that thresholding on the supports' areas simplifies textured zones, while retaining perceptually salient elements of the image.
\end{abstract}

Keywords: Mathematical morphology · Extinction values - Tree representation . Dynamics · Gray-scale thinnings

\section{Introduction}

Since their introduction by Vachier and Meyer [1], extinction values have been used as a measure of importance for the extrema of a signal [2,3]. When introduced, they were defined with respect to a granulometry; the extinction value of a maximum is the parameter of the smallest opening to completely erase it. The concept was then generalized [4]; for instance, volumic extinction values can be defined, although there is no corresponding granulometry.

While extinction values are typically used to select relevant markers, prior to watershed segmentation, this article introduces a new function decomposition associated to extinction values. In this article, we focus on a specific one, dynamics [5], but the idea can be easily extended to other ones.

The input signal can be written as a sum of simpler components that we will denote as main and secondary peaks, following a mountain climbing analogy. Moreover, the inclusions of the peaks' supports define a forest structure. This representation is different from state-of-the-art techniques such as the max-tree [6] or the tree of shapes [7].

Peaks are then attributed various measures of importance; removing or altering the least important peaks (in the sense of those criteria) leads to new operators, some of which we detail in this work. These operators differ from classic attribute openings or thinnings [8]. In particular, they do not obey the threshold decomposition principle [9]: we extend binary connected operators to gray-level operators by considering the supports of our peaks, instead of considering threshold sets.

All of the methodology presented in this article is applicable on signals defined on any finite simple graph; however, in the context of image processing, signals are usually 
defined on a regular grid in a two- or three-dimensional space. The decomposition that we introduce and all related operators have the additional attractive property of being invariant by isometries (rotations, translations, symmetries and combinations thereof).

\section{Notations}

In the following, $E$ denotes a finite, connected, simple graph. In most image processing applications, typically $E$ would be a subset of $\mathbb{Z}^{2}$ with a rectangular or hexagonal grid. For 3-D images, it could as well be a subset of $\mathbb{Z}^{3}$ with any arbitrary connectivity.

For a node $x \in E, N(x)$ denotes the neighborhood of $x$, i.e. all nodes $y \in E$ that share an edge with $x$.

The support of a function $f$ is the set of vertices on which it takes non-zero values: $\operatorname{supp}(f)=\{x \in E \mid f(x) \neq 0\}$.

\section{Decomposition in Main and Lesser Peaks}

In this section, we introduce a new function decomposition, that we call the decomposition in main and lesser peaks, by two different, equivalent ways of computing it. The first approach is based on successive geodesic reconstructions [10] from the global maxima of a signal - as this transformation in a way 'ignores' maxima of lesser values, which is in our case the desired behavior. The second approach is much more closely related to the theory of extinction values $[1,4]$, and although we focus here solely on dynamics [5], it can easily be extended to other extinction values.

\subsection{Decomposition via Successive Geodesic Reconstructions}

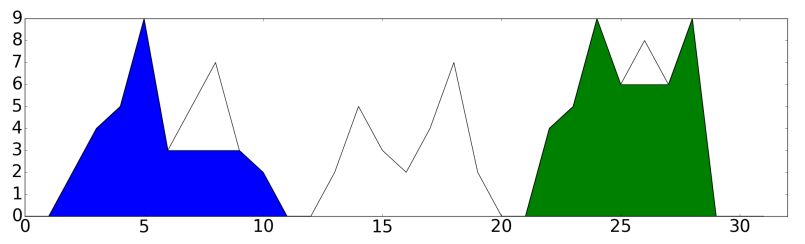

Fig. 1. Example of the elementary operation: the maximum of $f$ is $M=9$, reached at $X_{M}=$ $\{5,24,28\}$. There are two connected components in the support of the reconstruction $\Gamma(f)$, so we obtain two functions $f_{9}^{1}$ and $f_{9}^{2}$. One is shown in blue, the other in green.

Definition 1 (Geodesic reconstruction under the global maxima).

Let $f \in \operatorname{Fun}\left(E, \mathbb{R}^{+}\right), f \neq 0$.

Let $M=\max _{x \in E} f(x)$, and $X_{M}=\{x \in E \mid f(x)=M\}$ the set of points where it is reached.

We define $\Gamma(f)$ as the geodesic reconstruction of $f$ with markers $X_{M}$. 
Let $C^{1}, C^{2}, \ldots C^{K}$ be the $K$ connected components of $\operatorname{supp}(\Gamma(f))$, and for $1 \leqslant k \leqslant K$, let $f_{M}^{k}$ be the restriction of $\Gamma(f)$ on $C^{k}: f_{M}^{k}=\Gamma(f) \times \mathbb{1}_{C^{k}}$

In particular, $\Gamma(f)=\sum_{k=1}^{K} f_{M}^{k}$.

If $f$ is identically zero, we take $\Gamma(0)=0$ and the above summation is empty.

If $f$ is not identically zero, we call the $f_{M}^{k}$ the main peaks of function $f$.

Note that by construction, $\Gamma$ is an anti-extensive operator: $\Gamma(f) \leqslant f$, so the residue $f-\Gamma(f)$ is a nonnegative function, to which we can apply operator $\Gamma$ again. By iterating the process until the residue is zero, we obtain a first way of computing our decomposition (see algorithm 1).

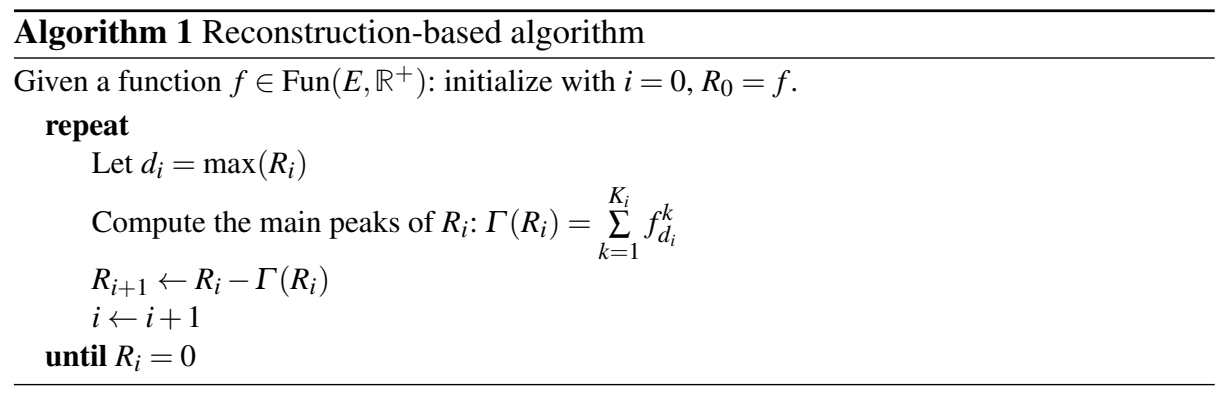

Let $I$ be the greatest integer such that $R_{I} \neq 0$; the previous algorithm yields our decomposition:

$$
f=\sum_{i=0}^{I} \sum_{k=1}^{K_{i}} f_{d_{i}}^{k}
$$

where, by construction, $d_{0}=M=\max f$.

Definition 2. We call the $f_{d_{0}}^{k}$ the main peaks of $f$, while for $i \geqslant 1$, we call the $f_{d_{i}}^{k}$ lesser peaks of $f$.

Figure 1 illustrates operator $\Gamma$ and the notion of main peaks.

It is easy to show that the previous algorithm converges in a finite number of steps: keeping the same notations as above, we have:

$$
\forall x \in X_{M}, \quad f(x)=\Gamma(f)(x)=M
$$

If $f \neq 0, X_{M} \neq \emptyset$ and the support of $f-\Gamma(f)$ is strictly included in the support of $f$. Since we chose $E$ to be a finite graph, the number of steps required for the algorithm to converge is upper-bounded by the cardinal of $E$.

Equation 1 also implies that the sequence $\left(d_{i}\right)_{1 \leqslant i \leqslant I}$ is strictly decreasing: $d_{0}>d_{1}>$ $\ldots>d_{I}$. 


\subsection{Decomposition via Razings}

The second way of computing the decomposition is quite similar to the watershed algorithm by flooding [11], except we raze the relief instead of flooding it. The idea is that we start from the regional maxima and gradually expand labels by following descending edges. When two or more regions meet, we stop propagating the labels that started from the lowest maxima, subtract the razed peak from the original function and propagate the remaining label. If two or more regions meet that started at the same highest - altitude, we merge the regions and replace their labels by a single one. This way of handling equality cases can lead to 'twin peaks', like the green component in figure 1, and it is debatable; many image processing algorithms would arbitrarily break such ties, considering for instance the lexicographical order on the coordinates when working on a rectangular subset of $\mathbb{Z}^{2}$, or the area extinction value, as was proposed by Grimaud [5]. The former approach is not invariant by rotation (swapping the coordinates will break ties in a different way) and is not applicable to the general case when the domain of definition is a finite simple graph; the latter is more attractive, but does not handle the case where the area extinction values are equal, too. Our way of handling ties ensures invariance by rotation when working on a regular grid, is well-defined, and as we shall see, yields interesting properties, in particular regarding the supports of the peaks.

Let us consider again a function $f \in \operatorname{Fun}\left(E, \mathbb{R}^{+}\right)$. If $f=0$ its decomposition in peaks is just an empty summation; in the following, let us assume $f$ is not identically zero and that its support is connected (if it is not, we simply apply the following procedure on each connected component).

Let us consider the set $X_{R M}$ of all regional maxima of $f$. A regional maximum of $f$ is a connected set $X \subset E$ such that $f$ takes the same value on each point of $X$ and stricly lower values on neighboring points of $X$.

Let $X_{R M}^{1}, \ldots X_{R M}^{K}$ be the $K$ connected components of $X_{R M}$, and let $L=\left\{l_{1}, \ldots, l_{L}\right\}=$ $f(E) \backslash\{0\}$ the set of values ('levels') taken by $f$ on its support, sorted in increasing order: $0<l_{1}<l_{2}<\ldots<l_{L}$.

The algorithm then goes as follows: we start by defining $K$ functions $f^{1}, \ldots, f^{K}$ which are simply the restrictions of $f$ on the connected components $X_{R M}^{1}, \ldots, X_{R M}^{K}$ :

$$
\forall k \in\{1, \ldots, K\}, f^{k}=f \times \mathbb{1}_{X_{R M}^{k}}
$$

We will then consider the connected components of the upper level sets of $f$, and progressively assign new points to one of the $f^{k}$ s until we have $f=\sum_{k=1}^{K} f^{k}$.

Let us denote by $F^{l}=\{x \in E \mid f(x) \geqslant l\}$ the upper set of function $f$ at level $l$; for each $i$ from $L$ to 1 , for each connected component $C$ of $F^{l_{i}}$, let us consider all $k$ 's such that $\operatorname{supp}\left(f^{k}\right) \subset C$ (there is at least one $\operatorname{such} k$ ).

There are two cases to consider: if only one of the $f^{k}$ has a maximum strictly greater than all others $\left(\max \left(f^{k}\right)>\max _{k^{\prime} \neq k} \max \left(f^{k^{\prime}}\right)\right)$, we consider it a potential main peak and assign to the others, which are necessarily secondary peaks, their final values: 


$$
\begin{array}{r}
\forall k^{\prime} \neq k:\left\{\begin{array}{c}
f^{k^{\prime}} \leftarrow f^{k^{\prime}}-l_{i} \\
\text { Finalize } f^{k^{\prime}}
\end{array}\right. \\
f \leftarrow f-\sum_{k^{\prime} \neq k} f^{k^{\prime}} \\
f^{k} \leftarrow f \times \mathbb{1}_{C}
\end{array}
$$

If there is only one $f^{k}$ whose support is a subset of $C$, obviously we would just assign it the value $f \times \mathbb{1}_{C}$. Else, if there is a tie between two or more peaks $\left(\max \left(f^{k_{1}}\right)=\right.$ $\left.\max \left(f^{k_{2}}\right)=\ldots=\max \left(f^{k_{m}}\right)=\max _{k} \max \left(f^{k}\right)\right)$, we first merge all these higher peaks together:

$$
\begin{aligned}
& f^{k_{1}} \leftarrow f^{k_{1}} \vee f^{k_{2}} \vee \ldots \vee f^{k_{m}} \\
& f^{k_{2}} \leftarrow 0 \\
& f^{k_{3}} \leftarrow 0 \\
& \ldots \\
& f^{k_{m}} \leftarrow 0
\end{aligned}
$$

then as before:

$$
\begin{aligned}
\forall k^{\prime} \notin\left\{k_{1}, k_{2}, \ldots, k_{m}\right\}: & \left\{\begin{array}{l}
f^{k^{\prime}} \leftarrow f^{k^{\prime}}-l_{i} \\
\text { Finalize } f^{k^{\prime}}
\end{array}\right. \\
& f \leftarrow f-\sum_{k^{\prime} \neq k_{1}} f^{k^{\prime}} \\
& f^{k_{1}} \leftarrow f \times \mathbb{1}_{C}
\end{aligned}
$$

In the previous instructions, 'finalize' $f^{k^{\prime}}$ means that when a peak $f^{k^{\prime}}$ is overtaken by a higher peak $f^{k}$ at level $l_{i}$, we subtract $l_{i}$ from it at this iteration, but then we will not change its value at any subsequent iteration.

After the last iteration, we have $f=\sum_{k=1}^{K} f^{k}$; this does not mean there are necessarily $K$ peaks, as some terms of the summation can be zero. After removing those, regrouping the terms with the same maximum value $d_{i}$ and reindexing, we can rewrite the summation as

$$
f=\sum_{i=0}^{I} \sum_{k=1}^{K_{i}} f_{d_{i}}^{k}
$$

as we had before. When we are not concerned with the values $d_{i}$, we may skip the double summation and write $f=\sum_{j=1}^{J} f^{j}$ for ease of reading.

Note that although this algorithm is mathematically valid for any finite simple graph $E$ and for any nonnegative real-valued function $f$, it can be more easily rewritten in the more restrictive - but more often encountered - case where $E$ is a regular grid and $f$ takes values in a finite set, typically $\{0, \ldots, 255\}$ for 8-bit images. Efficient algorithms exist for simulating floodings or razings, based on hierarchical queues [10]. Although this paper does not aim at providing a thorough algorithmic study, this remark means that computing the decomposition using this algorithm is roughly as time-expensive as computing the watershed transformation of an image. 


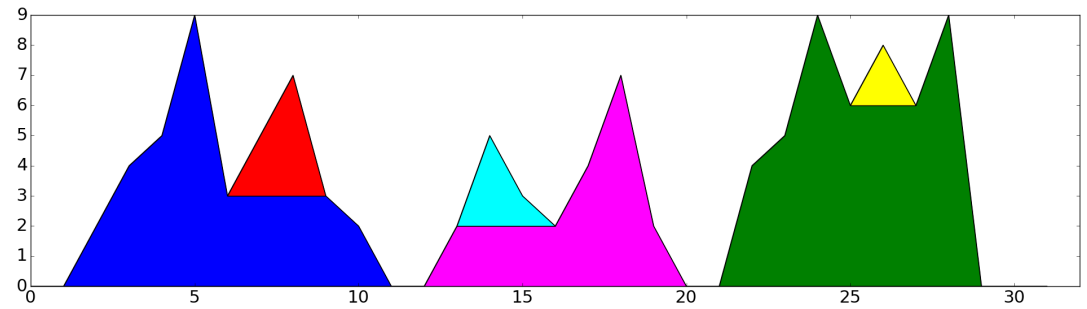

Fig. 2. Example of the decomposition

\subsection{Properties of the Decomposition}

Let $f=\sum_{i=0}^{I} \sum_{k=1}^{K_{i}} f_{d_{i}}^{k}$, as obtained by one of the previous algorithms, for a function $f: E \rightarrow$ $\mathbb{R}^{+}$.

Proposition 1 Nature of the peaks

Every peak $p$ obtained by our decomposition ( $p=f_{d_{i}}^{k}$ for some $i$ and $k$ ) satisfies the properties that:

1. $\operatorname{supp}(p)$ is connected

2. $\Gamma(p)=p$

Conversely, each function q satisfying these properties is by itself its own peak decomposition.

Proof. The first point is obvious by construction of the second algorithm. The fact that $\Gamma(p)=p$ comes from the fact that a function $f^{k}$ obtained by the second algorithm cannot have local maxima that are not global maxima, else they would have been assigned another component $f^{k^{\prime}}$.

The reciprocal sense is basically the definition given in the first algorithm.

We denote by $\mathbb{P}$ the set of functions from $E$ to $\mathbb{R}^{+}$satisfying those properties. In particular, peaks have no local maxima that are not global maxima.

A function $c: \mathbb{P} \rightarrow\{0,1\}$ will be referred to as an intrinsic criterion. In particular, any binary criterion [8] $T$ (defined not on the space of functions but on the space of subsets of $E$ ) can be associated a criterion $c_{T}$ on $\mathbb{P}$ by considering the support of a peak $p$ :

$$
c_{T}(p)=T(\operatorname{supp}(p)) .
$$

Proposition 2 If $x_{m}$ is the index of a local maximum of $f$ with dynamics $d$, then $x_{m}$ is the index of a global maximum of $f_{d}^{k}$ for some $k$, and $f_{d}^{k}\left(x_{m}\right)=d$.

The proof can be found in the original article defining the notion of dynamics [5], as the original proposed algorithm is similar to the second algorithm we propose; the originality of ours lies in the decomposition it computes along with the dynamics. 
A noticeable difference is how equality cases are handled: in [5], the author considers that two close maxima of the same value being assigned the same dynamics is undesirable behavior, since dynamics are often used as a way to select markers prior to segmentation, typically using the watershed algorithm. In this context, selecting many similar close extrema (usually minima, actually) is indeed undesirable behavior.

Since we chose not to break ties, our definition of the dynamics of a maximum is the one usually found in the literature: it is the difference in altitude between this maximum and the maximal lowest altitude of a point on a path joining it to another, strictly higher, maximum.

The dynamics of the global maximum (or global maxima) of a function $f$ is illdefined; the most common convention is to assign it the value $\max (f)-\min (f)$; other authors assign it an infinite value; if dynamics are to be used for ranking extrema by order of importance, the essential point is just to assign it a higher value than all local ones. In order to be consistent with the definitions of our algorithms, we will adopt the convention that the dynamics of the global maximum is simply its value. This is consistent with the fact that the value 0 plays a specific role in our decomposition. For instance, if the support of $f$ is not connected, $f$ and $f+\lambda$, where $\lambda>0$ may not have the same main peaks ( $f$ may have several, while $f+\lambda$ necessarily has only one). Following our mountain-climbing analogy, the value 0 can be thought of as the sea level; even if the support of $f$ is equal to the entire domain $E$, meaning that for all $x$ in $E, f(x)>0$, our reference level is not the minimum of $f$, but 0 .

Proposition 3 For any two components $f_{d_{i}}^{k}$ and $f_{d_{j}}^{k^{\prime}}, \operatorname{supp}\left(f_{d_{i}}^{k}\right)$ and $\operatorname{supp}\left(f_{d_{j}}^{k^{\prime}}\right)$ are either nested or disjoint.

In particular, if $\operatorname{supp}(f)$ is connected, then the number of main peaks $K_{0}=1$, $\Gamma(f)=f_{d_{0}}$ and:

$$
\forall i>1, \forall k \in\left\{1, \ldots, K_{i}\right\} \quad \operatorname{supp}\left(f_{d_{i}}^{k}\right) \subset \operatorname{supp}\left(f_{d_{0}}\right)
$$

This can be proved by induction; consider the razing-based algorithm: at the beginning, all supports are disjoint. Supports can only increase with iterations, and when peaks meet at level $l_{i}$, they are either merged together (if there is a tie) or some are finalized and the greater is assigned the value $l_{i}>0$ on the supports of the other.

Proposition 4 For any two components $f_{d_{i}}$ and $f_{d_{j}}$ :

$$
\operatorname{supp}\left(f_{d_{i}}\right) \subset \operatorname{supp}\left(f_{d_{j}}\right) \Rightarrow d_{i}<d_{j}
$$

Proposition 5 Stability by truncation

Let us consider a function $f \in \operatorname{Fun}\left(E, \mathbb{R}^{+}\right)$and its decomposition in main and lesser peaks $f=\sum_{i=0}^{I} \sum_{k=1}^{K_{i}} f_{d_{i}}^{k}$.

Let us now consider a function $g=\sum_{i=0}^{I} \sum_{k=1}^{K_{i}} \delta_{i}^{k} f_{d_{i}}^{k}$, where $\delta_{i}^{k} \in\{0,1\}$, that is, $g$ is a truncated summation of the components of $f$.

The peaks of $g$, as defined in the first section, are simply the peaks of $f$ that appear in the summation; the decomposition of $g$ can be written: 


$$
g=\sum_{i=0}^{I} \sum_{\substack{1 \leqslant k \leqslant K_{i} \\ \delta_{i}^{k}=1}} f_{d_{i}}^{k}
$$

In section 4 , we present several operators based on the idea of keeping only certain peaks, depending on certain criteria. The previous proposition asserts that, provided the criteria only depend on intrinsic properties (that is, the choice of $\delta_{i}^{k}$ only depends on $f_{d_{i}}^{k}$, these operators are idempotent.

\subsection{Induced Tree Structure}

Definition 3. If $\operatorname{supp}(f)$ is connected, then $\operatorname{supp}\left(f_{1}\right)=\operatorname{supp}(f)$, so proposition 3 naturally defines a tree structure on the components. Let $f_{d_{j}}$ be a child node of $f_{d_{i}}$ (we drop the superscripts for ease of reading) if:

$-\operatorname{supp}\left(f_{d_{j}}\right) \subset \operatorname{supp}\left(f_{d_{i}}\right)$

- There is no k such that $\operatorname{supp}\left(f_{d_{j}}\right) \subset \operatorname{supp}\left(f_{d_{k}}\right) \subset \operatorname{supp}\left(f_{d_{i}}\right)$

If $\operatorname{supp}(f)$ is not connected, the previous relationship yields a forest structure, with a tree for each connected component.

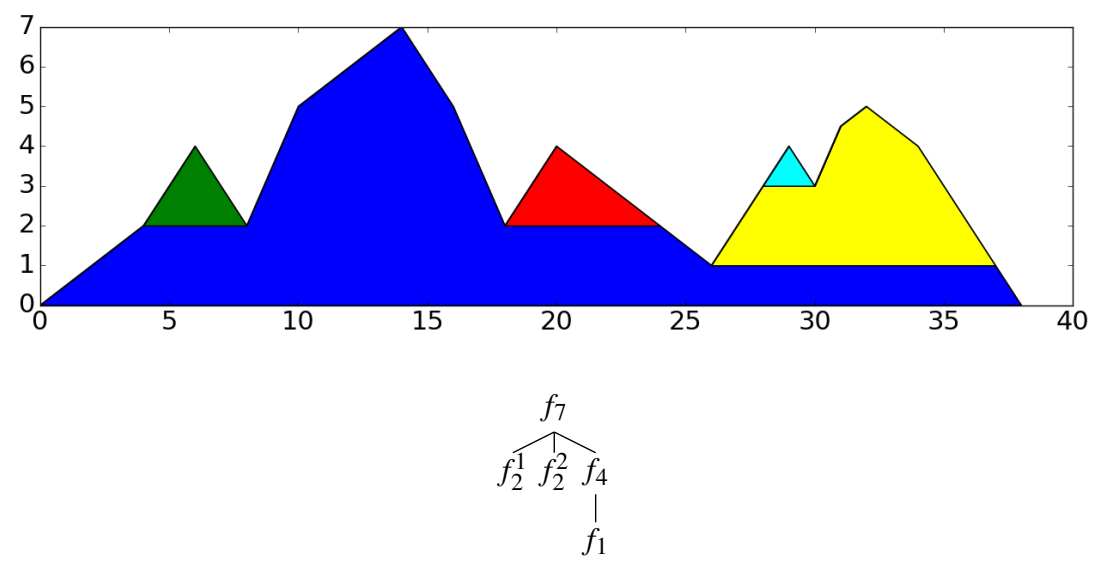

Fig. 3. Example of the decomposition and its associated tree: we have $f_{7}$ in blue, $f_{4}$ in yellow, $f_{2}^{1}$ and $f_{2}^{2}$ in green and red, $f_{1}$ in cyan.

Proposition 6 Stability by anamorphosis

The forest decomposition is stable by any nonnegative anamorphosis that goes through the origin; if $u$ is an increasing, invertible function from $\mathbb{R}^{+}$to $\mathbb{R}^{+}$with $u(0)=0$, then $f$ and $u(f)$ yield the same forest structure. 


\subsection{Ordering Induced by the Decomposition}

Let us consider $f$ and $g$ two functions from $E$ into $\mathbb{R}^{+}$, and their decompositions in main and lesser peaks, with the peaks labeled respectively from 1 to $J_{f}$ and from 1 to $J_{g}$ :

$$
f=\sum_{j=1}^{J_{f}} f^{j}, \quad g=\sum_{j=1}^{J_{g}} g^{j}
$$

We write $f \preceq g$ if there exists $\alpha:\left\{1, \ldots, J_{f}\right\} \rightarrow\left\{1, \ldots, J_{g}\right\}$ such that:

$$
\forall j \in\left\{1, \ldots, J_{g}\right\}, \sum_{i \mid \alpha(i)=j} f^{i} \leqslant g^{j}
$$

It can be easily seen that $f \preceq g \Rightarrow f \leqslant g$, but the reverse is not true in general.

Unfortunately, the couple $\left(\operatorname{Fun}\left(E, \mathbb{R}^{+}\right), \preceq\right)$ is not a lattice, nor even a semi-lattice: neither the infimum nor supremum of two functions are guaranteed to exist.

\section{Morphological Operators on $\left(\operatorname{Fun}\left(E, \mathbb{R}^{+}\right), \preceq\right)$}

In this section, we present some operators based on intrinsic criteria. All these operators are anti-extensive and idempotent; however, they lack the property of being increasing when considering the usual ordering on functions, so they are not morphological filters on the lattice $\left(\operatorname{Fun}\left(E, \mathbb{R}^{+}\right), \leqslant\right)$(they are only thinnings).

They are increasing, though, if considering the order $\preceq$ introduced in the previous section. It must be kept in mind that $\operatorname{since}\left(\operatorname{Fun}\left(E, \mathbb{R}^{+}\right), \preceq\right)$ is not a lattice, they stand outside the usual framework of mathematical morphology, but they can be thought of as morphological filters on a poset.

Definition 4. General form for criterion-defined operators

Given a function $f$, its peak decomposition

$$
f=\sum_{i=0}^{I} \sum_{k=1}^{K_{i}} f_{d_{i}}^{k}
$$

and an intrinsic criterion $c$, we define:

$$
\gamma_{c}:\left\{\begin{aligned}
\operatorname{Fun}\left(E, \mathbb{R}^{+}\right) & \rightarrow \operatorname{Fun}\left(E, \mathbb{R}^{+}\right) \\
f & \mapsto \sum_{i=0}^{I} \sum_{k=1}^{K_{i}} c\left(f_{d_{i}}^{k}\right) f_{d_{i}}^{k}
\end{aligned}\right.
$$

It is straightforward that any such operator is anti-extensive and idempotent; it is not, in general, increasing, be it for the usual order $\leqslant$ or the peak-induced order $\preceq$; we must add the constraint that the intrinsic criterion $c$ is itself increasing (for either order, as they are the same on $P$ ). Fortunately, this requirement is easily met. 
Definition 5. Peak dynamics thresholding

This tranformation has a parameter $\delta$, which is the minimum dynamics of peaks to be kept; the intrinsic criterion is a threshold above this value. Formally:

$$
\begin{aligned}
& c_{\delta}: P \rightarrow\{0,1\} \\
& p \mapsto\left\{\begin{array}{l}
1 \text { if } \max (p) \geqslant \delta \\
0 \text { else }
\end{array}\right.
\end{aligned}
$$

Definition 6. Peak area thresholding

This tranformation has a parameter A, which is the minimum area (cardinal of the support) of peaks to be kept; the intrinsic criterion is simply a threshold above this area. Formally:

$$
\begin{aligned}
c_{A}: P & \rightarrow\{0,1\} \\
p & \mapsto\left\{\begin{array}{l}
1 \text { if } \\
0 \text { else }
\end{array} \# \operatorname{supp}(p) \geqslant A\right.
\end{aligned}
$$

Definition 7. Peak volume thresholding

This time, the parameter $V$ is the minimal volume (sum of the values) of peaks to be kept; the intrinsic criterion is again a simple threshold above this parameter. Formally:

$$
\begin{aligned}
c_{A}: P & \rightarrow\left\{\begin{array}{l}
0,1\} \\
p
\end{array} \begin{array}{l}
1 \text { if } \\
0 \text { else }
\end{array} \sum_{x \in E} p(x) \geqslant V\right.
\end{aligned}
$$

These operators are illustrated in Fig. 4, in one dimension. Figure 5 illustrates peak area thresholding in two dimensions, and compares it to area opening, as defined by Vincent [12]. Gray-scale area opening razes all maxima until they become large enough flat zones, reducing the overall contrast of the image. Peak area thresholding, on the other hand, does not affect high or isolated maxima, thus preserving the most salient bright structures.

\section{Conclusions and perspectives}

In this article, we presented the methodology and mathematical foundations for obtaining a function decomposition based on a particular extinction value: dynamics. Instead of assigning a scalar value to each maximum, we assign it a function, that we call a peak, associated to this maximum. Those peaks can in turn be assigned different measures: maximum value, area, volume; binary criteria can also be computed on their supports. We exhibit some properties of our decomposition, and present a new order on functions induced by it.

Although we focused here on a dynamics-driven decomposition, similar decompositions can as easily be obtained by considering other extinction values in the definition of section 3.2. Area or volume extinction values will lead to different decompositions, and may be more sensible than dynamics, depending on the application and on the expected type of noise. 


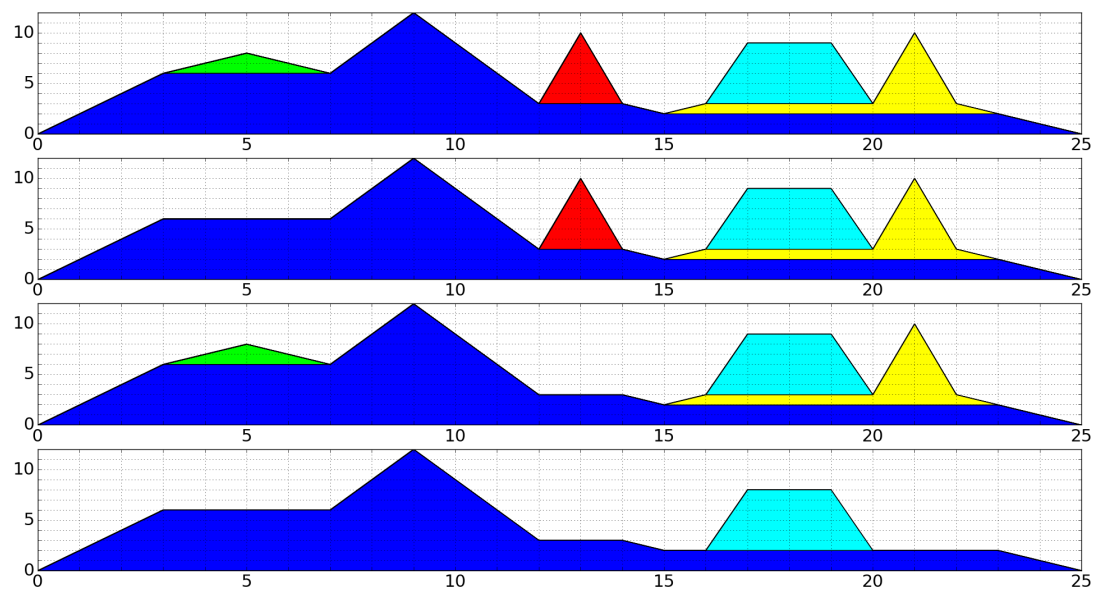

Fig. 4. From top to bottom: original signal, peak dynamics thresholding with $\delta=3$, peak area thresholding with $A=3$, peak volume thresholding with $V=15$.
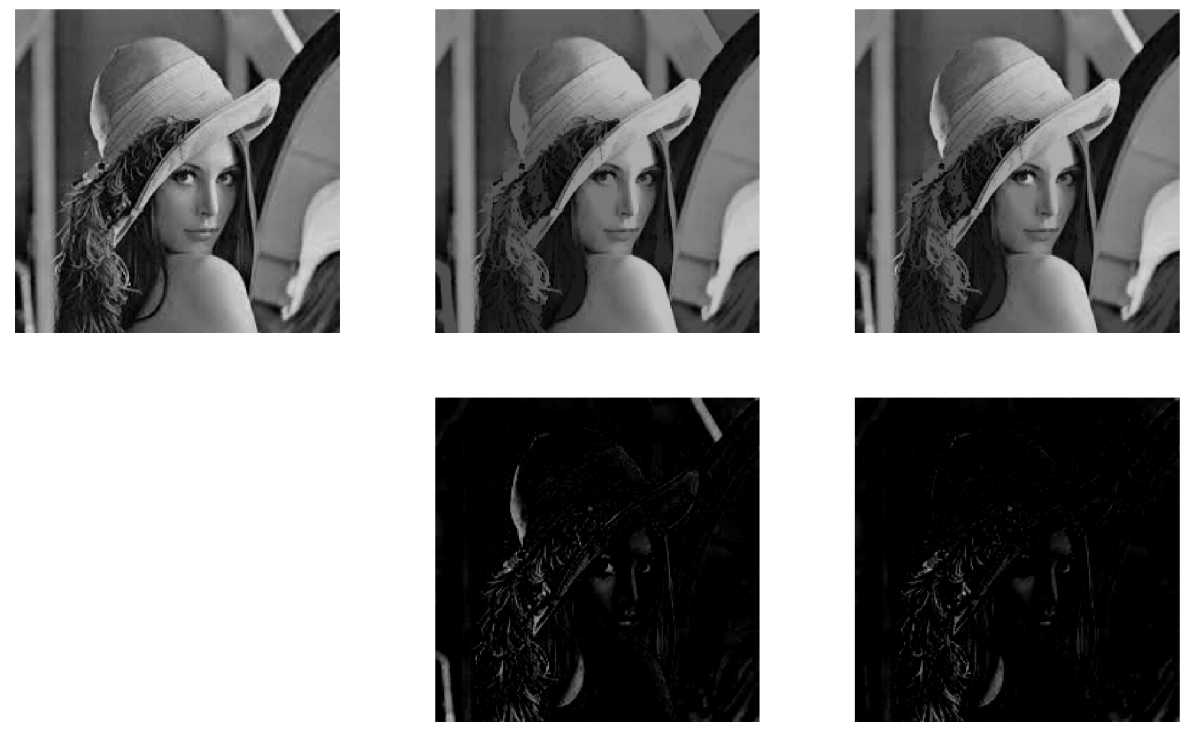

Fig. 5. Comparison between classical area opening and peak area thresholding; the original image is shown on the top left, the second column shows the result of a gray-scale area opening of size 350 and its residue; the rightmost column shows the result of a peak area thresholding of size 350 and its residue (the contrast of the residues has been enhanced for better visualization). We can see that peak area thresholding flattens textured zones, such as the hair, forehead and right cheek, but does not alter the most salient ones, such as the right eye or the reflection on the hat. 
In order to introduce the concepts, we considered nonnegative functions and the value zero as our reference level; however, it is easily generalized to real-valued functions by considering the positive and negative part and applying the decomposition to each, yielding self-dual operators.

\section{References}

[1] Corinne Vachier and Fernand Meyer. "Extinction value: a new measurement of persistence". In: IEEE Workshop on nonlinear signal and image processing. Vol. 1. 1995, pp. 254-257.

[2] Jesus Angulo and Jean Serra. "Automatic analysis of DNA microarray images using mathematical morphology”. In: Bioinformatics 19.5 (Mar. 2003), pp. 553562.

[3] Fernand Meyer and Corinne Vachier. "On the regularization of the watershed transform". In: Advances in imaging and electron physics 148 (2007), pp. 193249.

[4] Corinne Vachier. "Extraction de caractéristiques, segmentation d'image et morphologie mathématique". PhD thesis. École Nationale Supérieure des Mines de Paris, Dec. 1995.

[5] Michel Grimaud. "New measure of contrast: the dynamics". In: vol. 1769. International Society for Optics and Photonics. 1992, pp. 292-305.

[6] Philippe Salembier, Albert Oliveras, and Luis Garrido. "Antiextensive connected operators for image and sequence processing". In: Image Processing, IEEE Transactions on 7.4 (1998), pp. 555-570.

[7] Coloma Ballester, Vicent Caselles, and P. Monasse. "The tree of shapes of an image". In: ESAIM: Control, Optimisation and Calculus of Variations 9 (Jan. 2003), pp. 1-18.

[8] Edmond J. Breen and Ronald Jones. "Attribute openings, thinnings, and granulometries". In: Computer Vision and Image Understanding 64.3 (1996), pp. $377-$ 389.

[9] Henk J. A. M. Heijmans. "Theoretical aspects of gray-level morphology". In: IEEE Transactions on Pattern Analysis and Machine Intelligence 13.6 (1991), pp. 568-582.

[10] Serge Beucher. "Segmentation d'Images et Morphologie Mathématique". PhD thesis. Ecole Nationale Supérieure des Mines de Paris, 1990.

[11] Serge Beucher and Fernand Meyer. "The morphological approach to segmentation: the watershed transformation". In: Optical Engineering-New York-Marcel Dekker Incorporated 34 (1992), pp. 433-433.

[12] Luc Vincent. "Morphological area openings and closings for grey-scale images". In: Shape in Picture. Springer, 1994, pp. 197-208. 